\title{
Stability diagrams of colliding beams in the Large Hadron Collider
}

\author{
X. Buffat \\ CERN, 1211 Geneva, Switzerland and EPFL, 1015 Lausanne, Switzerland \\ W. Herr, N. Mounet, and T. Pieloni \\ CERN, 1211 Geneva, Switzerland
}

S. White

Brookhaven National Laboratory, Upton, New York 11973, USA

(Received 25 June 2014; published 25 November 2014)

\begin{abstract}
Most hadron synchrotrons rely on lattice nonlinearities for Landau damping of impedance driven coherent modes of oscillation. However, in a collider, the presence of beam-beam interactions strongly modifies the transverse amplitude detuning and therefore the resulting stability diagram. A numerical tool to evaluate the effect of beam-beam on the stability diagram has been developed and is used to discuss observations during different phases of the operational cycle of the Large Hadron Collider during the 2012 proton run. In particular, we show the evolution of the stability diagram when the strength of long range beam-beam interactions is increased, during the betatron squeeze. Also, we investigate the stability of beams colliding with a small transverse offset and compare to observations of instabilities when bringing the beams into collision and while leveling the luminosity.
\end{abstract}

DOI: 10.1103/PhysRevSTAB.17.111002

PACS numbers: 29.20.-c, 29.27.-a, 07.05.Tp

\section{INTRODUCTION}

In a synchrotron, the stability of coherent modes of oscillation driven by the beam coupling impedance is usually ensured by lattice nonlinearities, which cause particles at different betatron amplitude to oscillate at different frequencies, introducing Landau damping of coherent motion [1]. The strength of the Landau damping can be described by a stability diagram, which splits the complex plane in two parts determining the stability of a coherent mode characterized by its complex tune shift. Analytical derivations of the stability diagram can be found for simple configurations, e.g., considering linear detuning from octupolar magnets, assuming Gaussian or parabolic distribution functions [2,3]. In a collider, the amplitude detuning due to beam-beam interactions is usually comparable or larger than the one arising from lattice nonlinearities and therefore has to be taken into account in the computation of the stability diagram. An analytical form of the amplitude detuning can be found in special configurations, usually assuming few interaction points (IPs) with strong symmetry properties [4]. Yet, the analytical derivation of the dispersion integral is rather cumbersome in such cases. Also, CERN's Large Hadron Collider (LHC) features a complex configuration of multiple beam-beam interactions. Therefore, a fully

Published by the American Physical Society under the terms of the Creative Commons Attribution 3.0 License. Further distribution of this work must maintain attribution to the author(s) and the published article's title, journal citation, and DOI. numerical approach to compute both the amplitude detuning and the resulting stability diagram seems more appropriate. Following the approach developed in [5], we will derive stability diagrams in configurations relevant for the LHC operation, by evaluating the amplitude detuning with single particle tracking simulations and integrating numerically the dispersion integral. In Sec. II, we will describe the method and provide experimental justification for the assumptions made. In Sec. III, we apply the method to various configurations along the LHC operational cycle where beam-beam effects are significant, and compare to relevant observations of transverse instabilities.

In the following, we will refer to two different machine and beam configurations, which relevant parameters are summarized in Table I.

\section{METHOD}

Neglecting the coupling between the horizontal and vertical planes, as well as nonlinearities in the longitudinal

TABLE I. Beam and machine parameters in 2012 compared to nominal.

\begin{tabular}{lcc}
\hline \hline Parameter & Nominal & 2012 \\
\hline Energy [TeV] & 7 & 4 \\
Bunch intensity $\left[10^{11} \mathrm{p}\right]$ & 1.15 & 1.5 \\
Transverse rms emittance $[\mu \mathrm{m}]$ & 3.75 & 2.5 \\
Bunch spacing $[\mathrm{ns}]$ & 25 & 50 \\
$\beta^{*}[\mathrm{~m}]$ & 0.55 & 0.6 \\
Full crossing angle $[\mu \mathrm{rad}]$ & 285 & 290 \\
\hline \hline
\end{tabular}


degree of freedom, the stability diagram in each plane is obtained by solving the following dispersion relation for a given detuning $q\left(J_{x}, J_{y}\right)$ and distribution function $\psi\left(J_{x}, J_{y}\right)$ where $J_{x}$ and $J_{y}$ are the unperturbed action in each plane [2]:

$$
\begin{aligned}
\frac{-1}{\Delta Q_{i}} & =\iint_{0}^{\infty} \frac{J_{i} \frac{d \psi}{d J_{i}}}{Q-q\left(J_{x}, J_{y}\right)} d J_{x} d J_{y}, \\
Q & \in \mathbb{R}, \quad i=x, y .
\end{aligned}
$$

The tune shifts $\Delta Q_{i}$ found for different values of $Q$ are at the limit of stability, therefore, they define an area of the complex plane in which the tune shifts correspond to stable modes. When the denominator is strictly nonzero, the tune shift is purely real, which indicates the absence of Landau damping. Otherwise, the integrand exhibits some singularities that need to be properly addressed, as in [1] and references therein. Whereas difficult to obtain analytically in complex configurations, the detuning is easily obtainable numerically, via particle tracking. The dispersion integral can then be evaluated by standard numerical techniques, in our case by adding a small complex part to the denominator [6], between $i 10^{-6}$ and $i 5 \times 10^{-6}$, and using trapezoidal integration on a uniform grid [7] of $1000 \times 1000$ points with $J_{x}, J_{y} \in[0,18$ [. As a result of the finite upper limit in actions the contribution to the stability diagram of the far tails of the distribution is neglected. Considering Gaussian beam profiles, the upper limit was set at $6 \sigma$, the rms beam size, corresponding to the collimation range of the LHC [8].

In the multibunch regime, assuming that all bunches have an identical detuning, we obtain the same dispersion integral. However, in the LHC, the asymmetric layout of the IPs (Fig. 1) as well as the asymmetric filling scheme lead to a variety of bunches having significantly different number of head-on and long range beam-beam interactions [9], leading to different amplitude detuning. Theoretical treatment of the beam stability in such configuration is currently lacking, it is however possible to assess these cases using multiparticle tracking simulations [10], at the expense of large computational requirements.

The beam-beam interactions not only introduce bunch dependent detuning, they also change the nature of the coherent modes. The Landau damping of beam-beam modes in the single bunch regime is addressed in [11], the extension to multibunch coherent beam-beam mode is, however, not trivial. Studies by means of multiparticle tracking simulations are also presented in [10]. Such approach is well suited to study the LHC in its full complexity, however, it is very demanding in term of computing power, which render extended parametric studies difficult. Here we consider a simplified configuration, namely the stability of single bunch impedance modes, yet taking into account detuning from the lattice as well as beam-beam interactions. Multibunch effects from the impedance as well as beam-beam coherent modes are

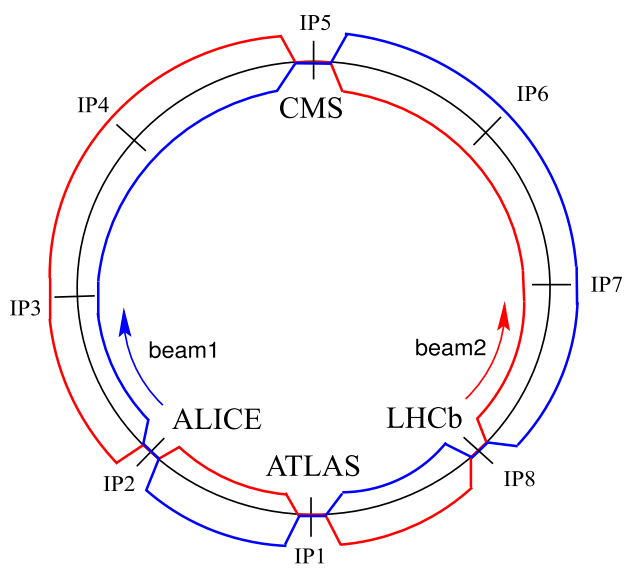

FIG. 1. Schematic layout of the IPs of the LHC.

neglected. These drastic assumptions are motivated by several observations, in the LHC, of single bunch instabilities, while operating in the multibunch regime. Figure 2 shows such an instability at the end of the betatron squeeze during operation of the LHC in 2012. In particular, the measurement of the beam oscillation amplitude provided by transverse pickups with direct diode detection, also known as the base band tune (BBQ) system [12], indicates a coherent instability on Beam 1. The observation of the bunch intensities indicates that only one bunch lost its intensity in an abnormal way, with respect to the other bunches, suggesting that this bunch only experienced the instability. This case is not isolated, most of the transverse instabilities observed during the operation of the LHC in 2011 and 2012 did not involve the full beam, but rather a subset of bunches. This feature of the instabilities is also discussed in [13].

In the following, the detuning is obtained numerically from tracking simulation with MAD-X $[14,15]$. Particles

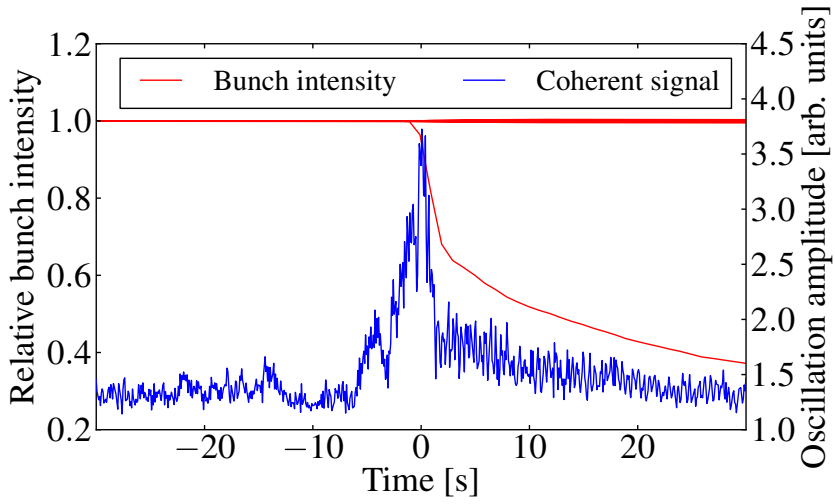

FIG. 2. Observation of an instability during luminosity production with 1380 bunches per beam. The beam oscillation amplitude in the horizontal plane of Beam 1 (in blue) shows a coherent excitation which is correlated with a significant intensity drop of a single bunch, as shown by the measured bunch by bunch intensities (in red). 


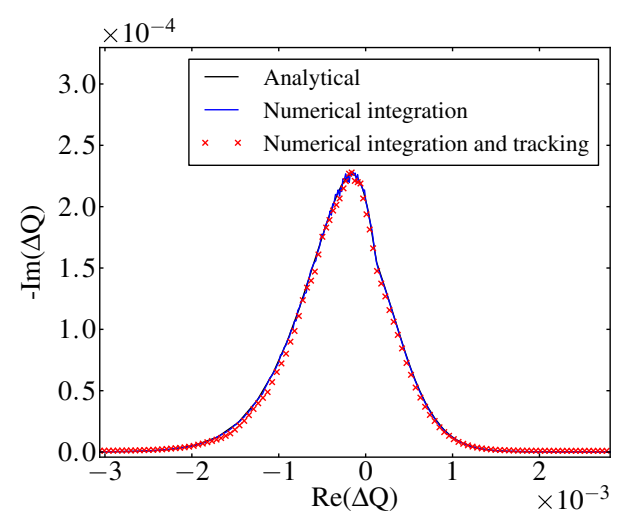

FIG. 3. Comparison of analytical [2] and numerical derivation of a stability diagram with LHC octupoles powered with $-500 \mathrm{~A}$ for a $4 \mathrm{TeV}$ beam with a normalized emittance of $2 \mu \mathrm{m}$.

with different actions are tracked for 1024 turns from which the tunes are computed using interpolated fast Fourier transform [16]. Also, a Gaussian distribution is always assumed. As shown in Fig. 3, there is a good agreement between the analytical and numerical approach in the case of octupolar detuning (Eq. 2).

\section{APPLICATIONS TO THE LHC OPERATIONAL CYCLE}

\section{A. Betatron squeeze}

The LHC beams share a common beam pipe only in a restricted area around each IP. Due to aperture considerations, the value of the $\beta$ function at the IP $\left(\beta^{*}\right)$ is kept significantly large during the injection of the beams into the machine, as well as the energy ramp. It is then decreased to its minimal value to maximize luminosity, through the socalled betatron squeeze. The strength of long range interactions mainly depends on the normalized separation, i.e., the distance between the beams, normalized to the beam size, at the location of each interaction. Before the squeeze, the normalized separation in the common area is in the order of $40 \sigma$, resulting in weak long range beambeam interactions. Thus, the amplitude detuning is dominated by the lattice nonlinearities, in particular due to octupole magnets used to provide Landau damping in the absence of beam-beam interactions [17]. Together with the transverse feedback, they ensure the stability of the beams. They can be powered with a current $I_{\text {oct }}$ up to $\sim 500 \mathrm{~A}$, with either polarity, giving rise to a linear amplitude detuning:

$$
\left\{\begin{array}{l}
\Delta Q_{x}=a \cdot J_{x}+b \cdot J_{y} \\
\Delta Q_{y}=b \cdot J_{x}+a \cdot J_{y}
\end{array},\right.
$$

where $J_{x}$ and $J_{y}$ are the transverse actions normalized to the beam normalized rms emittance $\epsilon$. The detuning coefficients for a beam energy of $E_{\text {beam }}$ are given by [17]:

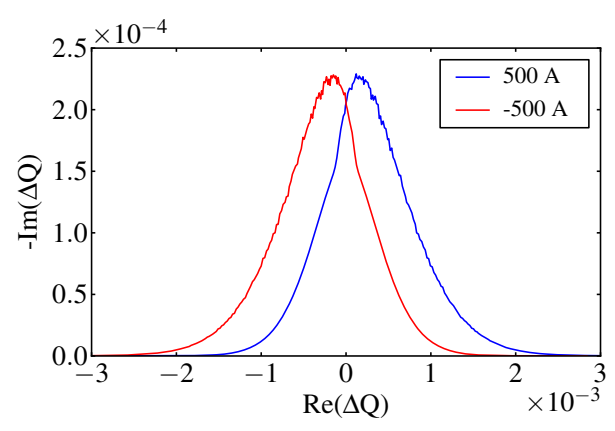

FIG. 4. Stability diagrams from octupoles powered with opposite polarities.

$$
\begin{aligned}
& a=3.28 \cdot \frac{I_{\mathrm{oct}}[\mathrm{A}] \cdot \epsilon[\mathrm{m}]}{E_{\text {beam }}^{2}\left[\mathrm{TeV}^{2}\right]} \\
& b=-2.32 \cdot \frac{I_{\text {oct }}[\mathrm{A}] \cdot \epsilon[\mathrm{m}]}{E_{\text {beam }}^{2}\left[\mathrm{TeV}^{2}\right]} .
\end{aligned}
$$

The resulting stability diagrams for each polarity are shown in Fig. 4. As the expected unstable modes in the LHC have tune shifts with negative real parts [18], the negative polarity is preferable in this configuration $[2,3]$. However, during the squeeze, the $\beta^{*}$ is reduced, while the crossing angle is kept constant, resulting in a smaller normalized separation at the location of each long range interaction. Thus, their effect starts playing a significant role in the single particle dynamics. As can be seen in Fig. 5, at the end of the squeeze, the normalized separation at the location of most of the long range interactions already has the value at which they will remain during luminosity production. The only difference being the parallel separation orbit bump, which affects only the interactions closest to the IP. As discussed in [19], this has a strong impact on the tune spread and consequently on the stability diagram. Figure 6 shows the modification of the stability diagram during the squeeze for two extreme bunches, one having the maximum number of long range interactions, referred to as common, and one with the least, referred to as PACMAN bunch [9]. With a negative polarity of the octupole, the long range contribution is partially

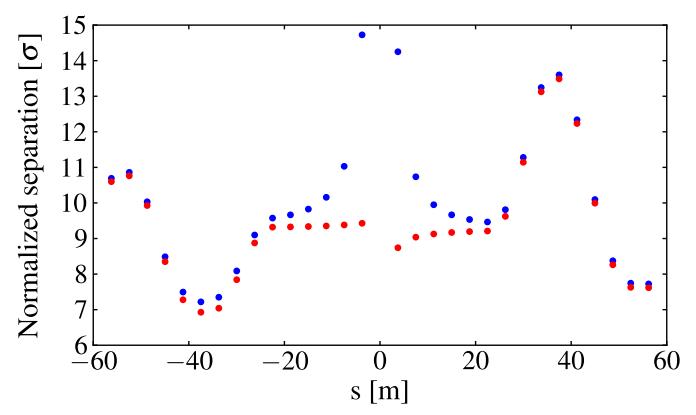

FIG. 5. Separation between the beams normalized to the beam size of Beam 1 at the location of the long range beam-beam interactions in the common chamber around IP5 at the end of the betatron squeeze (blue) and in collision (red). 


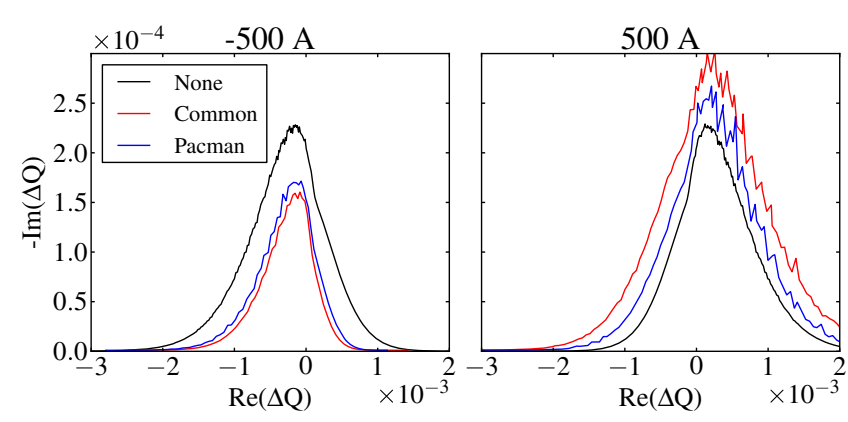

FIG. 6. Stability diagrams with both polarity of the octupoles for different long range contributions, corresponding to the configuration before the squeeze (None), after the squeeze, considering the most common bunch, i.e., largest long range contribution, and a PACMAN bunch with least long range contribution. The machine and beam parameters are those of 2012, neglecting the contribution of IP2 and 8.

compensating the octupoles detuning, resulting in a smaller stability diagram at the end of the squeeze. As a result, common bunches have a smaller stability diagram with respect to PACMAN bunches, all bunches being less stable after the squeeze with respect to before. The opposite is true with the positive polarity. Nevertheless, it is important to note that the most unstable bunch is the limitation for the operation of the machine, as the losses generated by a single bunch becoming unstable can trigger the machine protection system of the LHC, and therefore provoke a dump of the beams. Thus, in Fig. 7, we compare the smallest stability diagram in both configurations. One observes that, in the negative tune shift part, the stability diagram of PACMAN bunches in the case of $I_{\text {oct }}=500 \mathrm{~A}$, is very similar to the one of the most common bunch with $I_{\text {oct }}=-500 \mathrm{~A}$, in the configurations considered. Therefore, one should not expect a significant difference in the stability of the most critical bunches at the end of the squeeze with different polarities of the octupoles. This is consistent with observations of instabilities at the end of the squeeze in 2012 with both polarities of the octupoles. Nevertheless, there were no dedicated experiments to validate this statement and data acquired parasitically during standard operation are hardly interpretable.
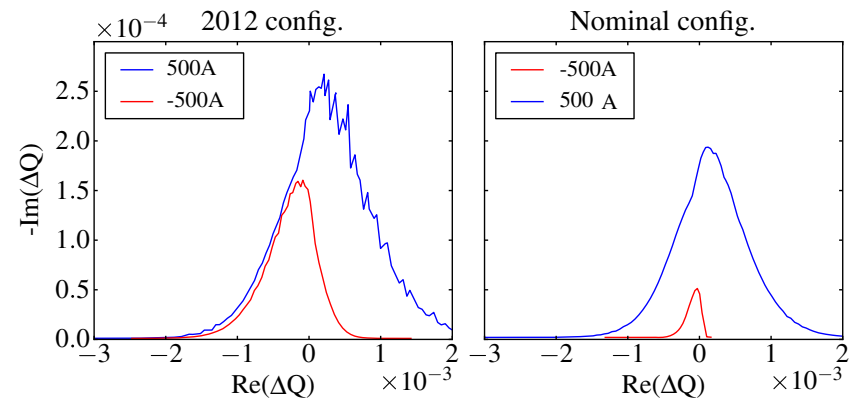

FIG. 7. Comparison of the worst bunch at the end of the squeeze for each polarity of the octupole, in 2012 and nominal configurations.

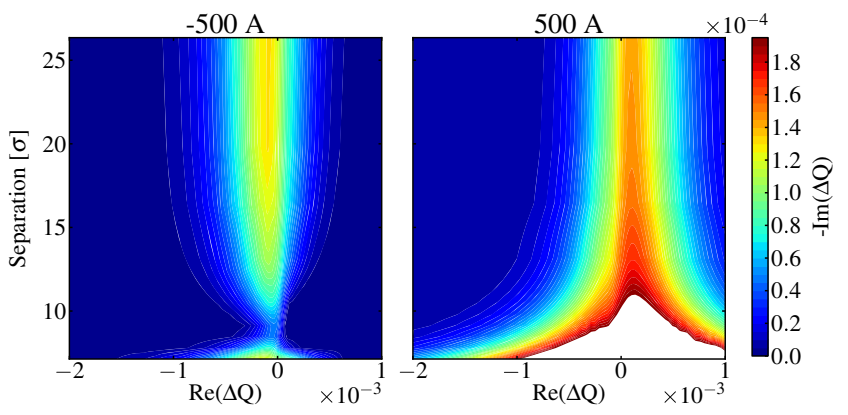

FIG. 8. Evolution of the most common bunch's stability diagram as the normalized separation between the beams is reduced. Nominal LHC beams have a normalized separation of $10 \sigma$ at the end of the squeeze.

Indeed, a single change of polarity was performed during the 2012 run, simultaneously to many other critical parameter changes. In particular, the chromaticity was raised from $\sim 2$ to $\sim 10-15$ units, significantly modifying the coherent dynamics, as discussed in [20], thus preventing proper comparison between the two configurations.

The LHC will restart with larger beam energy, 6.5 to $7 \mathrm{TeV}$, reducing the effectiveness of the octupoles, while keeping similar long range contributions. The effect of the compensation is therefore more important, with respect to 2012 configuration. As a result, the stability diagram at the end of the squeeze is much more critical, when using the negative polarity of the octupoles (Fig. 7).

Figure 8 illustrates the change from octupole dominated detuning to a configuration dominated by long range interactions by reducing the normalized separation at the location of the long range interaction. In the case of negative octupoles polarity, the transition results in a minimum of stability at a normalized separation of around $9 \sigma$ in the nominal configuration. This minimum is obtained for different normalized separation depending on the relative strength of the octupoles. In particular, in configurations with smaller emittances, the effectiveness of the octupoles is reduced, while the long range contributions do not change for identical normalized separation. As a result, the minimum of stability will be reached for larger separation, i.e., earlier in the squeeze. In such a configuration, the positive polarity, while less favorable before the squeeze, provides a larger stability diagram toward the end of the squeeze and may therefore be preferable.

As will be discussed later, the stability diagram due to head-on beam-beam interactions is significantly larger than the one provided by both long range and octupoles, therefore the considerations above no longer apply if head-on collisions are established before the execution of the squeeze.

\section{B. Bringing the beams into collision}

Collapsing the separation bump, in order to bring the beams into collision at the IP has two main impacts on the 


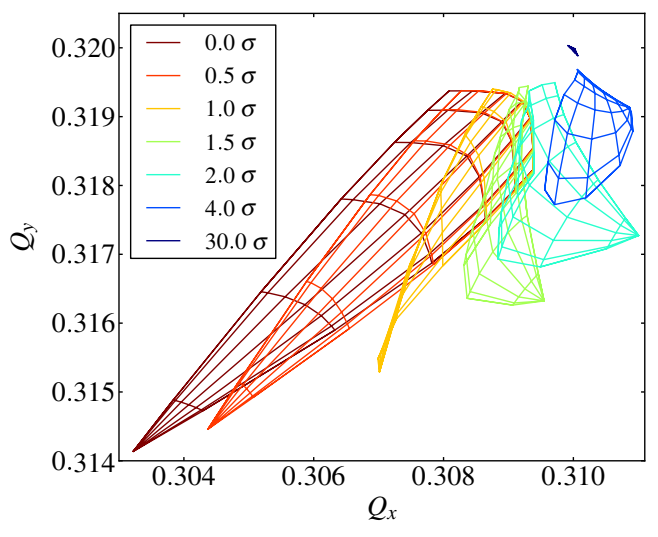

FIG. 9. Example of tune footprint of a bunch colliding in IP1 with different separations in the horizontal plane. The machine and beam parameters are those of 2012 (Table I).

stability diagram. First, as illustrated in Fig. 5, the separation at the location of the long range interaction is reduced. As a consequence, the effect of long range beam-beam interactions on the stability diagram, as described in Sec. III A, is increased during the process. Second, toward the end of the process, the tune shift and spread of the colliding bunches change sign as illustrated in Fig. 9, where the amplitude detuning is represented in a tune footprint, by reporting the horizontal and vertical tune of particles oscillating at different amplitudes into a tune diagram. This last part of the process represents a significant transition from a configuration where the single particle dynamic is mainly driven by the lattice nonlinearities and long range beam-beam interactions, to a regime dominated by the few beam-beam interactions at the IPs. As shown in Fig. 10, the stability diagram is enhanced for separations at the IP in the order of 2 to $4 \sigma$ and then

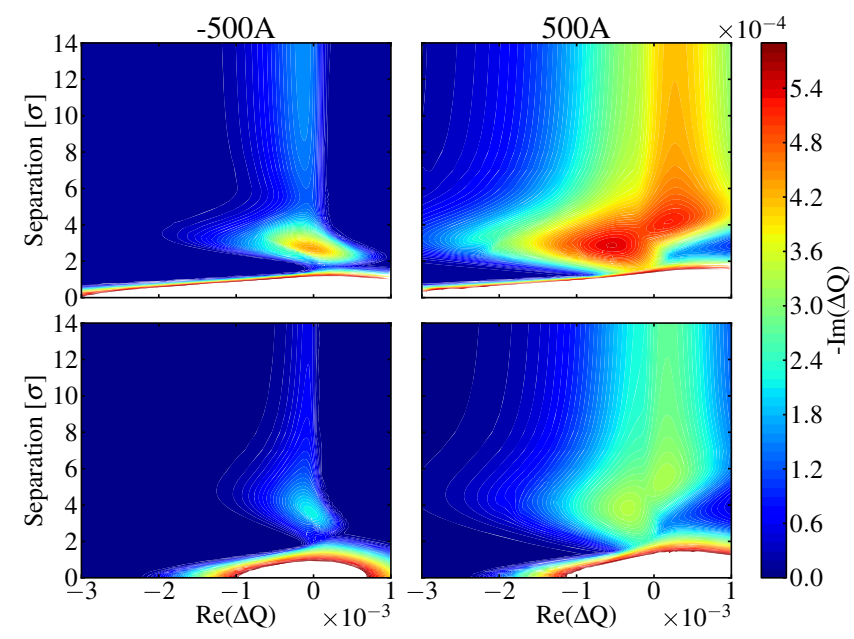

FIG. 10. Stability diagram as a function of beam separation in IP1 and 5 for both polarities of the octupoles. The upper and bottom plots corresponds to, respectively, 2012 and nominal configurations. drastically reduced around $1.5 \sigma$, leading to a significant modification of the stability diagram. This minimum of stability depends significantly on the configuration considered and therefore can be different for bunches with different number of long range or head-on interactions. As opposed to the interplay between octupoles and long range beam-beam interactions, the reduction of the stability diagram for beams colliding with a small transverse offset at the IP is not due to a compensation of tune spread. It is rather caused by a change of sign of the detuning of particles with a large amplitude, positive when dominated by long range and negative with head-on, which leads to a systematic cancellation of nearby poles in the dispersion integral. Yet, even if the minimum of stability exists independently of the polarity of the octupoles, it is clear from Fig. 10 that the positive polarity is also favorable in this configuration. Small variations are expected for bunches experiencing a different number of long range interactions.

Also, in Fig. 10, we considered an ideal process for the collapse of the separation, that is to say the separation in IP1 and 5 is collapsed synchronously in the horizontal and vertical plane, respectively. In such symmetric configurations, the behavior of the stability diagram as a function of the separation at the IP is identical in the two transverse planes. Yet, realistic configurations features an asynchronism of the orbit bumps in the two IPs, as well as a parasitic separation in the plane normal to the separation plane. These effects potentially break the symmetry between the two planes. A symmetric and an asymmetric configuration are compared in Fig. 11. One observes that, in the asymmetric configuration, the minimum of stability in the plane of the separation is similar to the symmetric configuration. While in the other plane, the minimum of stability is much less critical, and slightly asynchronous with respect to the other plane. The dispersion integral that we have used neglects the coupling between the transverse planes. However, when colliding with a small transverse offset, beam-beam interactions introduce strong linear and nonlinear coupling, which may play an important role in the beam stability. We shall illustrate this effect by the means of multiparticle tracking simulations. Coherent beam-beam modes are not considered in the model, the beam-beam force is, therefore, modeled statically, corresponding to the so-called weak-strong model. This multiparticle model features a consistent model of Landau damping with two transverse planes coupled by the beam-beam force. We perform the simulation with the code BEAMBEAM3D $[10,21]$ using a realistic model of the LHC impedance [18]. Figure 11 compares the growth rate of the most unstable mode as a function of the separation in the two configurations. We observe that, in the symmetric configuration, the beams are unstable for small separations, at the minimum of stability, whereas in the case where the symmetry between the planes is broken, 

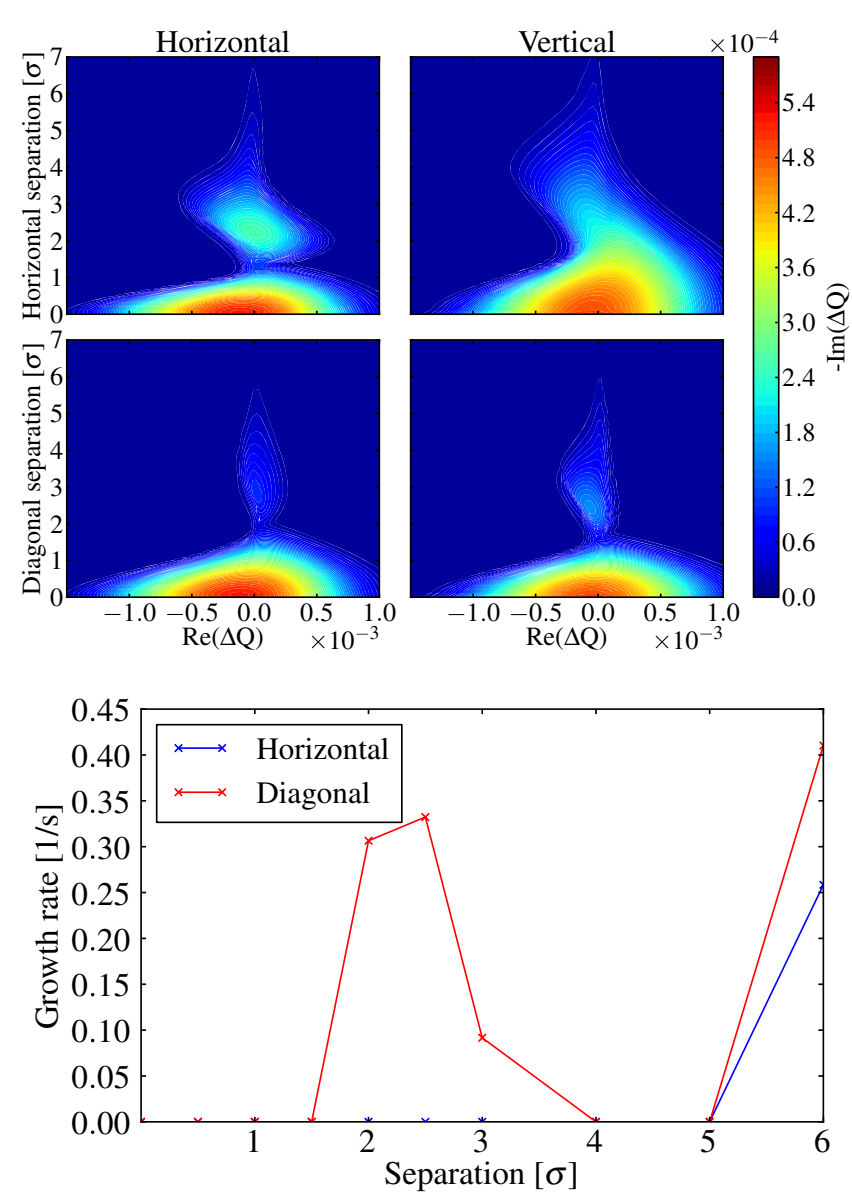

FIG. 11. Stability diagram (above) and simulated growth rate with weak-strong tracking (below), while collapsing the separation in one IP, in two configurations. In the first, the separation is in the horizontal plane, in the second on the diagonal, i.e., the separation is equal in the vertical and horizontal plane. The machine and beam parameters are those of 2012, without octupoles.

the beam is stable for separations below $5 \sigma$. Above this value, the beam is unstable in both configurations, as the tune spread due to the single beam-beam interaction becomes negligible at large separation.

Instabilities were observed when bringing the beams into collision during the first part of the year in 2012. The complexity of this process was significantly increased with respect to previous years, and with respect to the configuration discussed above, as it included a modification of the crossing in IP8 [22]. The length of the overall process was consequently increased. Figure 12 shows the separation in IP1 and IP5 in both planes during this process, which are to be compared with the histogram of 16 observations of coherent instabilities during this process and to the corresponding stability diagrams. These instabilities were observed with the negative polarity of the octupoles, the effect of long range interactions therefore reduces the stability diagram. Thus, during the first $40 \mathrm{~s}$ we observe a reduction of the stability diagram due to the collapse of
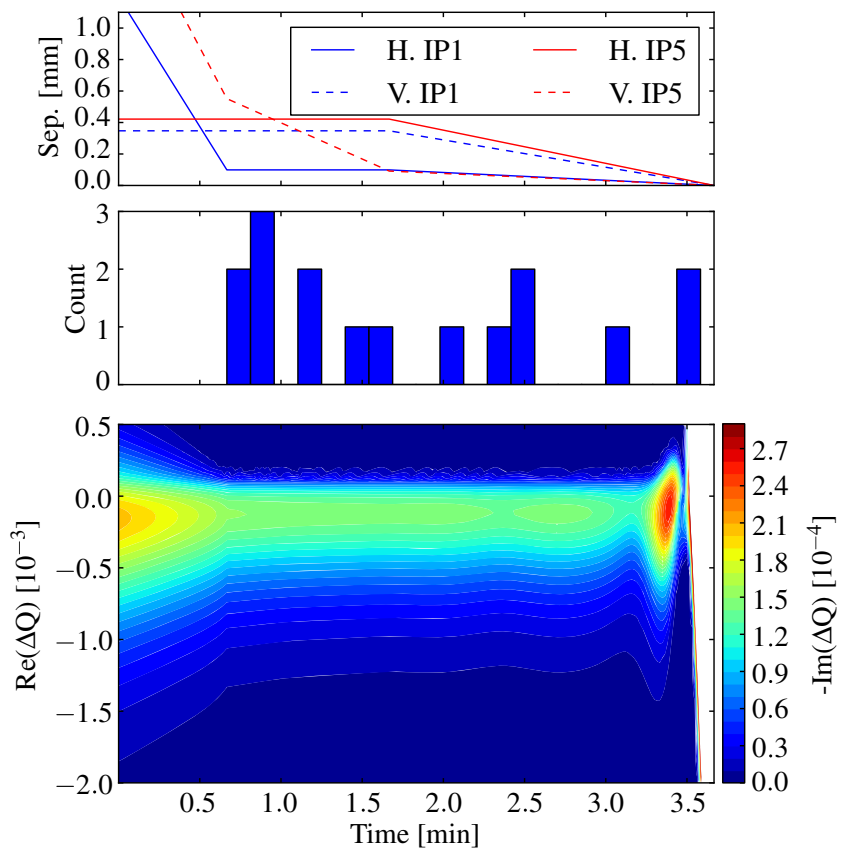

FIG. 12. The evolution of the separation in both planes in IP1 and IP5 (top), compared to the time at which the instabilities were observed during the process in the first part of 2012 (middle) and the stability diagram of the most common bunch during the procedure (bottom).

the separation bumps in IP1 and IP5. Except for the 2 occurrences few seconds before the end of the process, the instabilities are spread in the intermediate part of the process, during which the stability diagram remains constant. During this period, the separation in IP1 and IP5 are varied slowly such as to allow for the crossing angle change in IP8. These instabilities are therefore of the same nature as the ones at the end of the squeeze, i.e., the reduction of the stability diagram is the result of the interplay between the octupoles and long range beam-beam interactions. Such effect is not expected with the opposite polarity of the octupoles. As for the instabilities observed at the end of the squeeze, the operational data with the positive polarity of the octupole are hardly comparable, as the chromaticity was significantly different. Also, as this effect is driven by long range beam-beam interactions, it is not expected if the beams are brought into collision in a configuration where they are negligible, e.g., before the betatron squeeze.

The complication of the process introduced in order to allow for the crossing angle change in IP8 is not strictly necessary. Later in the year the process was reimplemented, separating the collapse of the separation bumps and the change of crossing angle in IP8. This allowed us to reduce the duration of the process from 3.6 down to 1.1 minutes. The speed of the process is critical, as a degradation of the beams due to a coherent stability is avoidable by going through critical phases on a shorter time scale. In the LHC, the expected rise times of impedance driven instabilities are typically in the order of few to several seconds $[10,18]$. 
Let us come back to the two instabilities near the end of the process, the separation at the IP may be estimated based on the luminosity measurement. In both cases, the separations in IP1 and IP5 are between 1 and $2 \sigma$, corresponding to the minimum of stability described in Fig. 10. Nevertheless, it was shown in [10] that beams colliding with a small transverse offset may exhibit strong mode coupling instability, which breaks the assumptions behind the dispersion integral. In the configuration considered, the coupling instabilities are expected around a separation of $1.5 \sigma$. The measurement of the beam oscillation amplitude by the BBQ shows activity in both beams simultaneously, suggesting that these instabilities are indeed due to coupling of impedance and beam-beam coherent modes. Nevertheless, the lack of diagnostics capable of measuring the correlation between the two beams prevents the conclusion of the exact nature of these instabilities.

\section{Luminosity production}

While colliding head-on, beam-beam is dominating the non-linearities experienced by the core of the beam and consequently provides the dominant contribution to the stability diagram. Figure 13 compares the tune footprints and resulting stability diagrams, generated by octupoles with positive polarity, long range and head-on, respectively. It is important to note that both the octupoles and long range have a large effect on the tails of the distribution. Due to the derivative of the distribution in the numerator of the dispersion integral [Eq. (1)], they yield a smaller stability diagram for a similar detuning. Therefore, the strength required in terms of octupoles or long range to obtain a similar stability diagram leads to an unacceptable reduction of the dynamic aperture.

The tune shift of most coherent modes lies inside the stability diagram provided by head-on collisions, therefore stabilization techniques required before bringing the beams into collision may no longer be required during luminosity production. However, specific requirements of each of the four experiments significantly increases the complexity [23]. The luminosity required in IP2 during
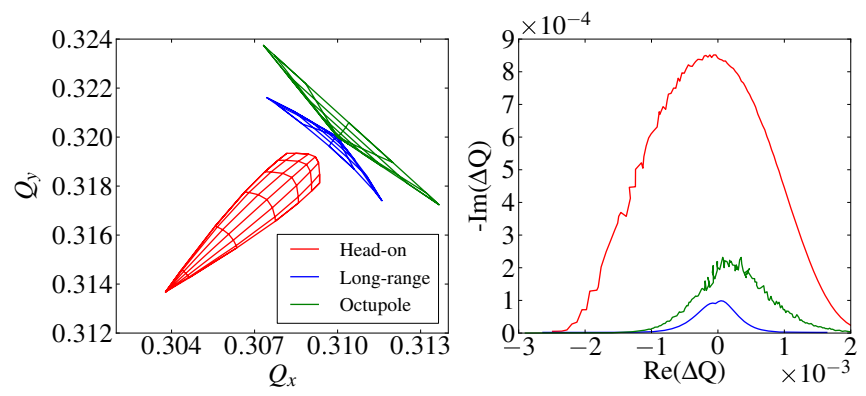

FIG. 13. Comparison of footprints and corresponding stability diagrams generated by octupoles powered with $+500 \mathrm{~A}$, long range in IP1 and 5 or head-on in IP1 and 5. The machine and beam parameters are those of the nominal configuration. proton physics is orders of magnitude lower than in the other experiments, which requires strong luminosity leveling, which leads to vanishing beam-beam forces. IP8 requires a smaller luminosity reduction factor and therefore the head-on contribution is not necessarily negligible. Yet, during the 2012 run, the luminosity was leveled with a transverse offset at the IP. The beams were therefore colliding strictly head-on only in the two high luminosity experiments located in IP1 and 5. Besides, these experiments have requested the presence of bunches without head-on collision in their experiment for background measurement purposes [23], resulting in a series of bunches having no head-on collision at all. As already mentioned, the stability of each bunch is crucial, which enforced the usage of strong stabilizing techniques, in particular high chromaticity, high transverse feedback gain and high current in the octupoles, during luminosity production in order to stabilize bunches without head-on collisions. While necessary for few bunches, these stabilizing techniques are potentially harmful for the intensity lifetime and emittance growth of the others. Ensuring at least one head-on collision for each bunch would allow us to relax the needs for stabilization and therefore allow for luminosity lifetime optimization.

There exist various ways to achieve this goal, which would fulfill the experiments' requests. One of them is the use of luminosity leveling with $\beta^{*}$ in IP8. Because of the small crossing angle, the tune shift due to head-on interaction is nearly independent of the $\beta^{*}$. Therefore, this technique has the advantage of providing the full head-on detuning all along the process of luminosity leveling. Thus, the configuration of bunches may be arranged such that all bunches collide head-on at least in one of the IPs 1,5 , or 8 .

\section{Leveling with a transverse offset}

During the 2012 run of the LHC, the luminosity was leveled with a transverse offset in IP8. While not harmful for most bunches with head-on interactions in IP1 and 5, this technique turned out to be critical for bunches without head-on collision in other experiments. Indeed, the situation of these bunches is similar to the one described in Fig. 10, the main difference with respect to the process that brings the beams into collision is the time scale. The separation is varied in small steps and several minutes are spent at each separation, leaving the time for a slow instability to develop. Observations of such instabilities during a fill dedicated to luminosity production are shown in Fig. 14. Many bunches experience an instability at the very beginning of luminosity production, during the adjustment of the orbit at the IP, while the separation is larger than $3 \sigma$. Yet, most bunches lose their intensities in a normal way during 5 hours of luminosity production and, suddenly, lose a significant fraction of their intensity on a time scale of a second. Simultaneously, a coherent signal is measured by 

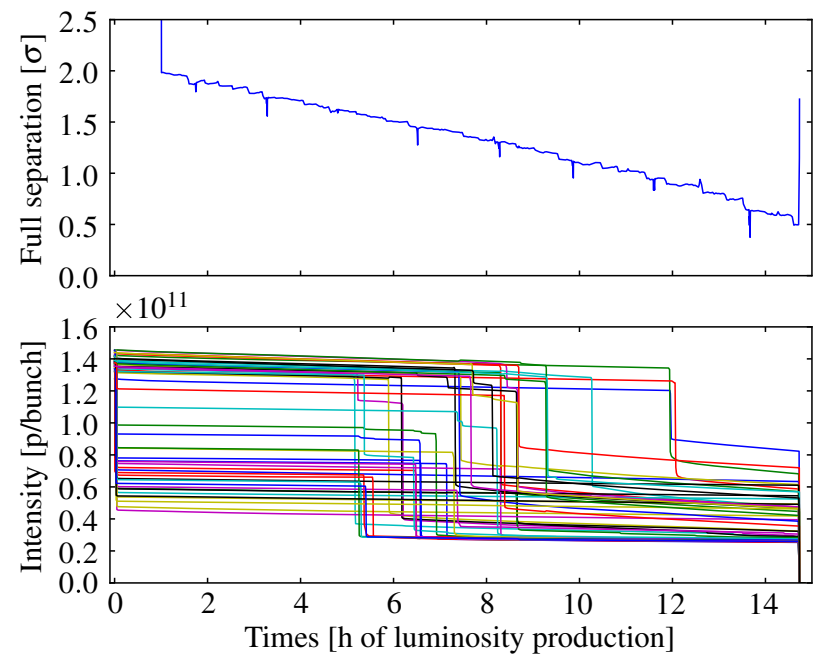

FIG. 14. Above, separation between the beams at the IP during luminosity leveling with a transverse offset in IP8, derived from measured luminosity in IP1,5 and 8, expressed in unit of the transverse beam size. Below, the intensity of the 49 bunches colliding only in IP8, the other 1331 bunches follow a standard quasiexponential decay.

the BBQ. This device does not, however, measure single bunch oscillation, the measurement of the rise times is therefore not possible.

The separation between the beams when the instabilities occurred is computed from measured total luminosities in IPs 1,5 and 8. Due to bunch to bunch variations of the emittance as well as the uncertainties on the $\beta^{*}$ in the different IPs, there are large uncertainties on the measured separation. We therefore combine the data of the fill analyzed in Fig. 14 with those of other similar fills using a kernel density estimation [24], i.e., the normalized addition of all measurements, each being modeled by a Gaussian distribution centered on the measured value and a width corresponding to the uncertainty (Fig. 15). It appears

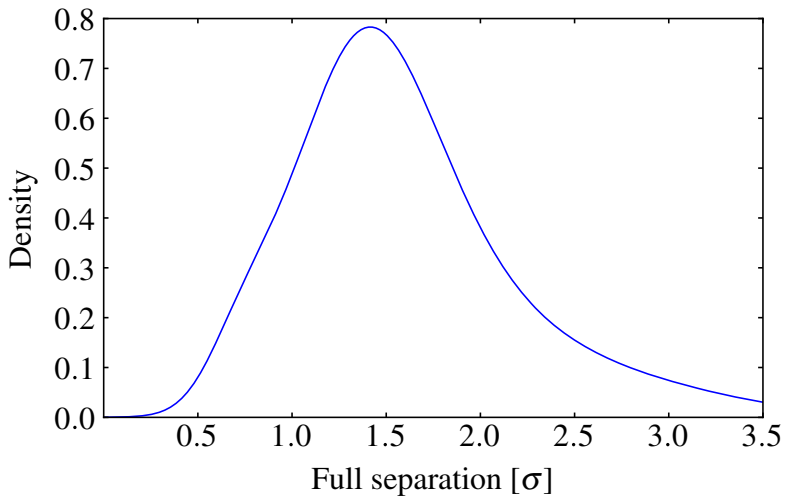

FIG. 15. Kernel density estimation of the separation between the beams at the moment of the instability. 30 occurrences are considered with an uncertainty of $24 \%$ on the measured separation, accounting for bunch to bunch variations of the emittance (10\%) as well as for the error on the $\beta^{*}$ at the IPs 1,5 , and 8 [25]. that the full separation in IP8 at the time of the instabilities was between 0.9 and $1.6 \sigma$, consistent with the critical separation discussed previously. The statistics is biased due to the leveling range, which typically started between 2 and $3 \sigma$, and, apart from few exceptions, stopped before reaching $1 \sigma$. Nevertheless, the narrowness of the peak suggests that there indeed exists a critical separation around $1.5 \sigma$. It is difficult to make quantitative comparison with predictions for each individual observation of instability as many critical parameters are not known to a sufficient precision, in particular each bunch emittance. Yet, we may understand the fact that the bunches become unstable at slightly different separations. First, in the configuration of bunches used during these fills, the bunches colliding only in IP8 had each a different number of long range interactions, resulting in slightly different stability diagrams. Second, there were bunch to bunch variations of the intensity and emittances in the order of $10 \%$.

As opposed to the instabilities observed towards the end of the process that brings the beams into collision, no coherent signal is observed in the other beam. This excludes the coupled coherent beam-beam modes as an explanation for these instabilities [26]. The subtle difference between these two configurations is that the bunches colliding in IP8 with those experiencing the instability do collide head-on in IP1 and IP5. These collisions provide sufficient stability for the other beam, neglecting the coherent contribution of beam-beam interactions is therefore a good approximation in this configuration.

\section{SUMMARY AND OUTLOOK}

A numerical integrator was used to evaluate the dispersion integral using amplitude detuning evaluated with single particle tracking with MAD-X. This method allows us to derive stability diagram in different operational phases of the LHC, including its full complexity, in particular beam-beam interactions. It was shown that, due to the increase of the strength of long range interactions during the betatron squeeze, the stability diagram is significantly enhanced or reduced, in case the octupoles are powered with, respectively, positive or negative polarity.

The stability was shown to be critical with beams colliding with a small transverse offset, in accordance with observations made bringing the beams into collision, and leveling luminosity with a transverse offset. While avoidable by bringing the beams into collision faster than the rise times of the expected instabilities, this kind of instability is difficult to avoid when leveling luminosity with a transverse offset. In such configuration, the usage of other stabilizing techniques is necessary.

It was shown that head-on collisions, acting on core particles rather than tail, are very efficient in providing Landau damping. 


\section{ACKNOWLEDGMENTS}

The authors would like to acknowledge G. Arduini, B. Salvant, E. Métral, S. Redaelli, and J. Wenninger for fruitful collaborations and discussions, as well as the LHC operation crew, in particular G. Papotti and R. Giachino for their work. This work is partially supported by Brookhaven Science Associates, LLC under Contract No. DE-AC02-98CH10886 and LARP with the U.S. Department of Energy. This research used resources of the National Energy Research Scientific Computing Center, which is supported by the Office of Science of the U.S. Department of Energy under Contract No. DEAC02-05CH11231.

[1] D. Sagan, Am. J. Phys. 62, 450 (1994).

[2] J. Scott Berg and F. Ruggiero, CERN Technical Report No. CERN SL-AP-96-71, 1996.

[3] E. Métral and A. Verdier, CERN Technical Report No. CERN-AB-2004-019-ABP, 2004.

[4] A. W. Chao, Report No. SLAC-PUB-9574, 2002.

[5] W. Herr and L. Vos, LHC Project Note 316, 1998.

[6] G. Korn and T. Korn, Mathematical Handbook for Scientists and Engineers: Definitions, Theorems, and Formulas for Reference and Review, 2nd ed., (Dover Publications, Inc, Mineola, 2000) Chap. 7.7.

[7] K. Atkinson, An Introduction to Numerical Analysis, 2nd ed., (John Wiley \& Sons, Inc, New York, 1989) Chap. 5.1.

[8] R. Assmann et al., in Proceedings of the 46th ICFA Advanced Beam Dynamics Workshop on High-Intensity and High-Brightness Hadron Beams, edited by A. Adelman, J. Chrin, M. Marx, V. Schaa, and M. Seidel (JACoW, Geneva, Switzerland, 2011), p. 21.

[9] W. Herr, LHC Project Note 628, 2003.
[10] S. White, X. Buffat, N. Mounet, and T. Pieloni, Phys. Rev. ST Accel. Beams 17, 041002 (2014).

[11] Y. Alexahin, Nucl. Instrum. Methods Phys. Res., Sect. A 480, 253 (2002).

[12] M. Gasior and R. Jones, LHC Project Report 853, 2005.

[13] A. Burov, Phys. Rev. ST Accel. Beams 17, 021007 (2014).

[14] http://cern.ch/mad.

[15] W. Herr, LHC Project Note 344, 2004.

[16] R. Bartolini and F. Schmidt, CERN Report No. Note 98017, 1998.

[17] J. Gareyte, J.-P. Koutchouk, and F. Ruggiero, LHC Project Report 91, 1997.

[18] N. Mounet, Ph.D. thesis, EPFL, 2012.

[19] S. Fartoukh, Presentation at the LHC Machine Committee, 2012.

[20] E. Métral, in Proceedings of Chamonix 2011 Workshop on LHC Performance, Chamonix, France, 24-28 January 2011, edited by C. Carli (CERN, Geneva, Switzerland, 2011), pp. 252-260.

[21] J. Qiang, M. A. Furman, R. D. Ryne, W. Fischer, and K. Ohmi, Nucl. Instrum. Methods Phys. Res, Sec. A 558, 351 (2006).

[22] B. Holzer, in Proceedings of the LHC Beam Operation Workshop (CERN, Geneva, 2012).

[23] R. Jacobsson, in Proceedings of the ICFA Mini Workshop on Beam-Beam Effects in Hadron Colliders, Geneva, Switzerland, 18-22 March 2013, edited by W. Herr and G. Papotti (CERN, Geneva, Switzerland 2014), pp. 231-236.

[24] B. Sliverman, Density Estimation for Statistics and Data Analysis (Chapman \& Hall, London, 1986).

[25] R. Tomás, T. Bach, R. Calaga, A. Langner, Y. I. Levinsen, E. H. Maclean, T. H. B. Persson, P. K. Skowronski, M. Strzelczyk, G. Vanbavinckhove, and R. Miyamoto, Phys. Rev. ST Accel. Beams 15, 091001 (2012).

[26] X. Buffat, Ph.D. thesis, EPFL, 2014. 